\title{
Combination of Interacting Multiple Models with the Particle Filter for Three-Dimensional Target Tracking in Underwater Wireless Sensor Networks
}

\author{
Xin Wang, ${ }^{1}$ Mengxi Xu, ${ }^{2}$ Huibin Wang, ${ }^{1}$ Yan $W u^{1}$ and Haiyan Shi ${ }^{3}$ \\ ${ }^{1}$ College of Computer and Information, Hohai University, Nanjing, Jiangsu 211100, China \\ ${ }^{2}$ School of Computer Engineering, Nanjing Institute of Technology, Jiangsu 210000, China \\ ${ }^{3}$ College of Computer Science and Technology, Zhejiang University of Technology, \\ Zhejiang 310023, China
}

Correspondence should be addressed to Xin Wang, wang_xin@hhu.edu.cn

Received 4 October 2012; Accepted 13 November 2012

Academic Editor: Sheng-yong Chen

Copyright (C) 2012 Xin Wang et al. This is an open access article distributed under the Creative Commons Attribution License, which permits unrestricted use, distribution, and reproduction in any medium, provided the original work is properly cited.

\begin{abstract}
Tracking underwater targets is a focused application area in modern underwater defence systems. Using traditional techniques based on imaging or sensor arrays may be difficult and impractical in some mission-critical systems. Alternatively, the underwater wireless sensor network (UWSN) is able to offer a promising solution. This paper tackles the problem of accurately tracking underwater targets moving through the UWSN environment. This problem is considered nonlinear and non-Gaussian where the present solution methods based on the particle filter technique are powerful and simple to implement. For three-dimensional underwater maneuvering target tracking, the traditional particle filter tracking algorithm may fail to track the targets robustly and accurately. Thus, the interacting multiple model method is combined with the particle filter to cope with uncertainties in target maneuvers. Simulation results show that the proposed method is a promising substitute for the traditional imaging-based or sensor-based approaches.
\end{abstract}

\section{Introduction}

Nowadays, tracking targets in the underwater environment is an indispensable part in many military or civil fields, such as modern underwater defense systems, navigation and control of mobile robots, and traffic monitoring in intelligent transportation systems. Much attention has been paid to it and various techniques have been developed based on imaging [1] or sensor arrays $[2,3]$. Thereinto, imaging-based methods utilize underwater optical imaging or underwater sonar imaging for underwater target tracking. However, for the imaging process is always disturbed by water turbidity, underwater illumination intensity, or underwater 
noise, such techniques usually cannot get satisfactory tracking results. Other underwater target tracking techniques use underwater sensor arrays to measure azimuth, elevation angle, and distance of the underwater targets and then track them by classical filtering techniques, such as Kalman's filter (KF), extended Kalman filter (EKF), and unscented Kalman filter (UKF) [4]. Nevertheless, these sensor arrays are generally mounted on or towed by a ship or a submersible or deployed prior to the application, which makes the tracking range limited or makes them unsuitable for on-demand tracking missions [5].

In terms of surveillance and reconnaissance, the wireless sensor network (WSN) stands as a promising technology [6-8]. The main advantages of WSN include its low cost, rapid deployment, self-organization, and fault tolerance [9]. As a specific case of WSN, the underwater wireless sensor network (UWSN) is developed to enable applications for assisted navigation, oceanographic data collection, obstacle avoidance, and tactical surveillance [10]. Some effort has been made for underwater target tracking with UWSN [11-13]. For example, in [11], Yu et al. present a distributed tracking algorithm for a single moving target through UWSN as well as the issues of estimating the state of target and improving energy efficiency by applying a Kalman filter in a distributed architecture. In [12], Isbitiren and Akan design a three-dimensional (3D) underwater target tracking algorithm for underwater acoustic sensor networks. Based on the time of arrival of the echoes from the target after transmitting acoustic pulses from the sensors, the ranges of the nodes to the target are determined, and trilateration is used to obtain the location of the target. The location and the calculated velocity of the target are then exploited to achieve tracking. Kim et al. [13] propose a bearings-only tracking scheme based on distributed floating beacons in UWSN. It adopts a multidimensional assignment for rejecting clutters and unscented Kalman filter for tracking a target.

To achieve three-dimensional (3D) underwater target tracking, the following challenges must be considered. For example, the measurement value of the sensor usually has a nonlinear relationship with the estimated value in UWSN. However, most of the existing algorithms for underwater target tracking do not consider the nonlinear problem, which results in poor tracking accuracy. Moreover, in order to avoid underwater obstacles or other moving objects, the trajectory of the underwater target might be very complex. A single motion model is not sufficient to capture its movement.

To solve the aforementioned issues, we present combination of interacting multiple model (IMM) with the particle filter (PF) for three-dimensional target tracking in UWSN. The particle filter [14-17] is a sequential Monte Carlo approach that uses random samples, called particles, to approximate the posterior probability density. It is an efficient way to solve nonlinear and non-Gaussian problems [18-20]. However, the traditional PF algorithm with a single motion model cannot solve the maneuvering problems of underwater target tracking [21]. For maneuvering target tracking, the interacting multiple model (IMM) method is widely used $[22,23]$. It can estimate the state of a dynamic system with several behavioral modes that switch from one to another using mode likelihoods and mode transition probabilities. In the beginning, the filter underlying the modes in the IMM framework was KF or EKF. Later, PF was substituted for KF or EKF in the IMM framework (so-called IMMPF) [24]. Subsequently, IMMPF is widely applied to various target tracking problems [25, 26]. For instance, in [25], the IMMPF is applied to the problem of maneuvering target tracking with passive coherent location radar. In [26], it is used for a land vehicle Global Position System/Dead Reckoning navigation system. Although IMMPF has been extensively used, the study of target tracking in UWSN is still in its infancy. Therefore, this paper focuses on the issue of underwater target tracking based on IMMPF. 
The rest of the paper is organized as follows. In Section 2, the UWSN architecture and target motion models, as well as the measurement model of the UWSN are given. Section 3 presents the IMMPF 3D underwater target tracking algorithm. Following that, simulation results are presented in Section 4, and conclusions are drawn in Section 5.

\section{Problem Formulation}

\subsection{UWSN Architecture}

Figure 1 shows the architecture of the UWSN used for 3D underwater target tracking. It is composed of multiple underwater acoustic sensors. These sensor nodes are deployed by binding to ropes which are docked onto the bed of water or a floater. As a result, all nodes form a three-dimensional network. In this paper, we consider three different types of sensor nodes: surface buoys, anchor nodes, and ordinary nodes. Surface buoys are nodes that drift on the water surface. They are often equipped with common Global Positioning System (GPS) and can get their absolute locations from GPS. Anchor nodes are those who can directly contact the surface buoys to get their absolute positions. Ordinary nodes cannot directly talk to the surface buoys because of cost or other constraints but can communicate with anchor nodes to estimate their own positions. There are extensive studies for the localization of UWSN [27-29]. In this paper, we will not contribute to this part. Instead, we mainly tackle the problem of underwater target tracking with UWSN.

\subsection{Underwater Target Motion Models}

For underwater target tracking, to precisely predict and estimate the motion information of targets, the exact motion models of targets should be designed [30-33]. In order to capture the movement of the underwater target, three different dynamic models, namely, constant velocity $(\mathrm{CV})$, constant acceleration (CA), and coordinated turn (CT), are studied here.

Underwater target motion can be described by the following discrete dynamic state equation:

$$
\mathbf{X}_{k+1}=\mathbf{F}_{k} \mathbf{X}_{k}+\mathbf{w}_{k}
$$

where $\mathbf{X}_{k}$ is the target state vector at the $k$ time step; $\mathbf{F}_{k}$ is the state transition matrix; $\mathbf{w}_{k}$ is the process noise.

Considering that the underwater target moves in a three-dimensional physical world, the target state vector $\mathbf{X}_{k}$ can be defined as

$$
\mathbf{X}_{k}=\left[\begin{array}{lllllllll}
x_{k} & \dot{x}_{k} & \ddot{x}_{k} & y_{k} & \dot{y}_{k} & \ddot{y}_{k} & z_{k} & \dot{z}_{k} & \ddot{z}_{k}
\end{array}\right]^{T},
$$

where $x_{k}$ is the position of the target along $x$-axis at $k ; \dot{x}_{k}$ is the $x$-axis velocity of the target at $k$; $\ddot{x}_{k}$ is the $x$-axis acceleration of the target at $k . y_{k}, \dot{y}_{k}, \ddot{y}_{k}, z_{k}, \dot{z}_{k}$, and $\ddot{z}_{k}$ are similarly defined. Notice that the acceleration of the target at each coordinate equals to zero for CV model and the state vector is taken to be $\mathbf{X}_{k}=\left[\begin{array}{cccccc}x_{k} & \dot{x}_{k} & y_{k} & \dot{y}_{k} & z_{k} & \dot{z}_{k}\end{array}\right]^{T}$. 


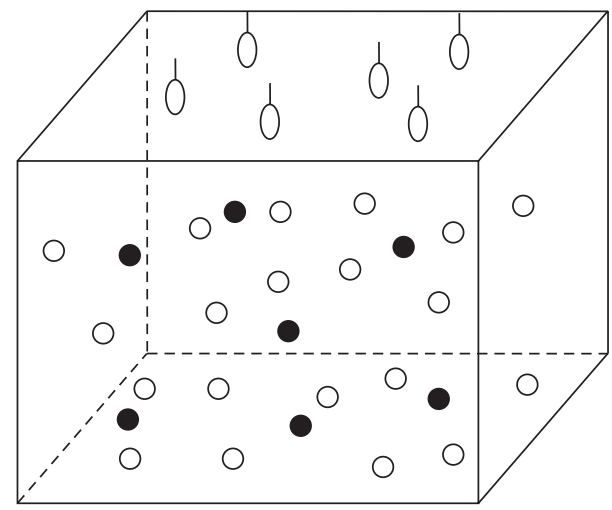

$\oint$ Surface buoy

- Anchor node

Ordinary node

Figure 1: Architecture of UWSN.

\subsubsection{Model}

The constant velocity model is the basic motion manner for underwater targets, such as submarines, torpedoes, and autonomous underwater vehicles. The state transition matrix $\mathbf{F}_{k}$ of this model is

$$
\mathbf{F}_{k}=\left[\begin{array}{llllll}
1 & T & 0 & 0 & 0 & 0 \\
0 & 1 & 0 & 0 & 0 & 0 \\
0 & 0 & 1 & T & 0 & 0 \\
0 & 0 & 0 & 1 & 0 & 0 \\
0 & 0 & 0 & 0 & 1 & T \\
0 & 0 & 0 & 0 & 0 & 1
\end{array}\right],
$$

where $T$ is the time interval between samples.

While the underwater target does constant velocity motion, its speed may change slightly due to the effect of wind and water flow, which can be represented by a white noise process $\mathbf{w}_{k}$ with variance $\sigma_{w}^{2}$. The corresponding covariance matrix of the noise is given by

$$
\mathbf{Q}_{k}=\left[\begin{array}{cccccc}
\frac{T^{4}}{4} & \frac{T^{3}}{2} & 0 & 0 & 0 & 0 \\
\frac{T^{3}}{2} & T^{2} & 0 & 0 & 0 & 0 \\
0 & 0 & \frac{T^{4}}{4} & \frac{T^{3}}{2} & 0 & 0 \\
0 & 0 & \frac{T^{3}}{2} & T^{2} & 0 & 0 \\
0 & 0 & 0 & 0 & \frac{T^{4}}{4} & \frac{T^{3}}{2} \\
0 & 0 & 0 & 0 & \frac{T^{3}}{2} & T^{2}
\end{array}\right] \sigma_{w}^{2} .
$$




\subsubsection{CA Model}

Beside the nonmaneuvering motion, we need to consider maneuvering motions for underwater targets. To account for the accelerations in the movement, the constant acceleration model is introduced. Its state transition matrix $\mathbf{F}_{k}$ and the covariance matrix $\mathbf{Q}_{k}$ of the independent white noise process $\mathbf{w}_{k}$ are

$$
\begin{aligned}
& \mathbf{F}_{k}=\left[\begin{array}{ccccccccc}
1 & T & \frac{T^{2}}{2} & 0 & 0 & 0 & 0 & 0 & 0 \\
0 & 1 & T & 0 & 0 & 0 & 0 & 0 & 0 \\
0 & 0 & 1 & 0 & 0 & 0 & 0 & 0 & 0 \\
0 & 0 & 0 & 1 & T & \frac{T^{2}}{2} & 0 & 0 & 0 \\
0 & 0 & 0 & 0 & 1 & T & 0 & 0 & 0 \\
0 & 0 & 0 & 0 & 0 & 1 & 0 & 0 & 0 \\
0 & 0 & 0 & 0 & 0 & 0 & 1 & T & \frac{T^{2}}{2} \\
0 & 0 & 0 & 0 & 0 & 0 & 0 & 1 & T \\
0 & 0 & 0 & 0 & 0 & 0 & 0 & 0 & 1
\end{array}\right], \\
& \mathbf{Q}_{k}=\left[\begin{array}{ccccccccc}
\frac{T^{4}}{4} & \frac{T^{3}}{2} & \frac{T^{2}}{2} & 0 & 0 & 0 & 0 & 0 & 0 \\
\frac{T^{3}}{2} & \frac{T^{2}}{2} & T & 0 & 0 & 0 & 0 & 0 & 0 \\
\frac{T^{2}}{2} & T & 1 & 0 & 0 & 0 & 0 & 0 & 0 \\
0 & 0 & 0 & \frac{T^{4}}{4} & \frac{T^{3}}{2} & \frac{T^{2}}{2} & 0 & 0 & 0 \\
0 & 0 & 0 & \frac{T^{3}}{2} & \frac{T^{2}}{2} & T & 0 & 0 & 0 \\
0 & 0 & 0 & \frac{T^{2}}{2} & T & 1 & 0 & 0 & 0 \\
0 & 0 & 0 & 0 & 0 & 0 & \frac{T^{4}}{4} & \frac{T^{3}}{2} & \frac{T^{2}}{2} \\
0 & 0 & 0 & 0 & 0 & 0 & \frac{T^{3}}{2} & \frac{T^{2}}{2} & T \\
0 & 0 & 0 & 0 & 0 & 0 & \frac{T^{2}}{2} & T & 1
\end{array}\right] \sigma_{w}^{2} .
\end{aligned}
$$

\subsubsection{CT Model}

Sometimes, the underwater target may make a turn. In order to capture this behavior, the coordinated turn model is investigated. This model presumes that the target moves with 
constant speed and constant turn rate. Assuming that the underwater target turns only in the $\mathrm{XY}$-plane, the corresponding state transition matrix $\mathbf{F}_{k}$ is

$$
F_{k}=\left[\begin{array}{ccccccccc}
1 & \frac{\sin \omega T}{\omega} & 0 & 0 & -\frac{1-\cos \omega T}{\omega} & 0 & 0 & 0 & 0 \\
0 & \cos \omega T & 0 & 0 & -\sin \omega T & 0 & 0 & 0 & 0 \\
0 & 0 & 1 & 0 & 0 & 0 & 0 & 0 & 0 \\
0 & \frac{1-\cos \omega T}{\omega} & 0 & 1 & \frac{\sin \omega T}{\omega} & 0 & 0 & 0 & 0 \\
0 & \sin \omega T & 0 & 0 & \cos \omega T & 0 & 0 & 0 & 0 \\
0 & 0 & 0 & 0 & 0 & 1 & 0 & 0 & 0 \\
0 & 0 & 0 & 0 & 0 & 0 & 1 & 0 & 0 \\
0 & 0 & 0 & 0 & 0 & 0 & 0 & 1 & 0 \\
0 & 0 & 0 & 0 & 0 & 0 & 0 & 0 & 1
\end{array}\right],
$$

where $\omega$ is the turn rate. The zero-mean white noise $\mathbf{w}_{k}$ in the CA model is used to model the perturbation of the trajectory from the CT motion.

\subsection{Measurement Model of UWSN}

Sensors used for target tracking provide measurements of a target, such as range $r$, azimuth $\alpha$, and elevation angle $\beta$ [31]. For example, there are $N=3$ sensor nodes involved in target tracking (see Figure 2). $\left(x_{k}, y_{k}, z_{k}\right)$ are the coordinates of the target at time $k .\left(x_{i}, y_{i}, z_{i}\right)$ represent the coordinates of the $i$ th sensor node. The measurements $r_{k, i}, \alpha_{k, i}$, and $\beta_{k, i}$ represent the distance, azimuth, and elevation angle provided by the $i$ th sensor node, respectively. equations:

From Figure 2, we can see that the measurements can be calculated by the following

$$
\begin{gathered}
r_{k, i}=\sqrt{\left(x_{k}-x_{i}\right)^{2}+\left(y_{k}-y_{i}\right)^{2}+\left(z_{k}-z_{i}\right)^{2}}, \quad i=1,2, \ldots, N, \\
\alpha_{k, i}=\arctan \frac{y_{k}-y_{i}}{x_{k}-x_{i}}, \quad i=1,2, \ldots, N, \\
\beta_{k, i}=\arctan \frac{z_{k}-z_{i}}{\sqrt{\left(x_{k}-x_{i}\right)^{2}+\left(y_{k}-y_{i}\right)^{2}}}, \quad i=1,2, \ldots, N .
\end{gathered}
$$

The measurement equation from the $i$ th sensor is given by

$$
\begin{aligned}
\mathbf{Z}_{k, i} & =\mathbf{h}_{k, i}\left(\mathbf{X}_{k}\right)+\mathbf{v}_{k, i} \\
& =\left[r_{k, i}, \alpha_{k, i}, \beta_{k, i}\right]^{T}+\mathbf{v}_{k, i}, \quad i=1,2, \ldots, N,
\end{aligned}
$$




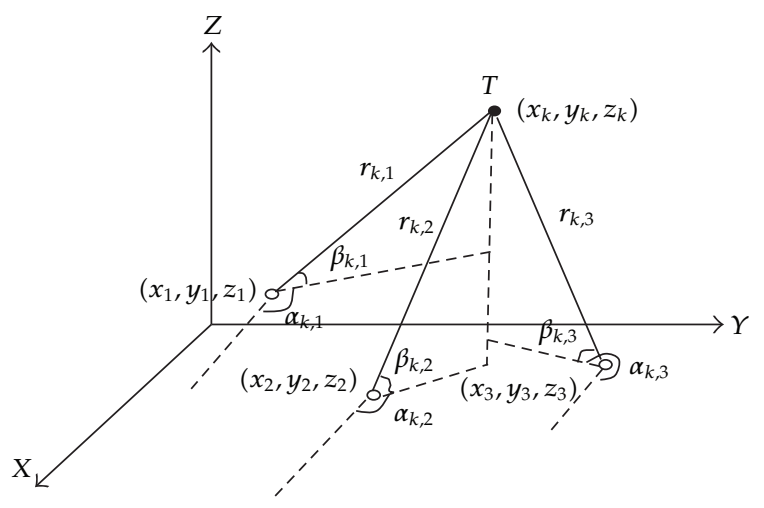

Figure 2: Measurement system of UWSN.

where $\mathbf{Z}_{k, i}$ is the measurement from the $i$ th sensor at time $k . \mathbf{v}_{k, i}$ is the independent zeromean Gaussian measurement noise with covariance matrix $\mathbf{R}_{k}$. At time $k$, the accumulated measurement of $U W S N Z_{k}$ is given by

$$
\mathbf{Z}_{k}=\left[\mathbf{Z}_{k, 1}, \mathbf{Z}_{k, 2}, \ldots \mathbf{Z}_{k, N}\right]^{T}
$$

\section{IMMPF Underwater Target Tracking Algorithm}

\subsection{Interacting Multiple Model}

The interacting multiple model, as one of the most efficient dynamic multiple model (MM) estimators, was proposed by Blom and Bar-Shalom [34]. Different from many other methods which assume a particular moving pattern of the node, the IMM filter incorporates all the possible moving patterns of the node, by running a bank of filters parallel with each filter corresponding to one particular moving pattern. And the overall state estimate is a certain combination of these model-conditional estimates. A complete cycle of the IMM filter process consists of four essential operations, namely, mixing/interaction, filtering, mode probability update, and combination [35]. The flow diagram of an IMM method is shown in Figure 3.

\subsection{IMMPF}

Particle filter can solve the nonlinear problem of underwater target tracking. However, it cannot handle the maneuvers of the target for it only uses a single motion model. The interacting multiple model method is suitable for tracking maneuvering motions of the target. Therefore, we combine IMM with PF, namely, IMMPF, for three-dimensional target tracking in UWSN. The flowchart of IMMPF algorithm is shown in Figure 4. It can be noted that IMMPF has four stages: mixing and interaction stage, particle filter stage (emphasized by the dotted box), mode probability update stage, and state update stage (combination). 


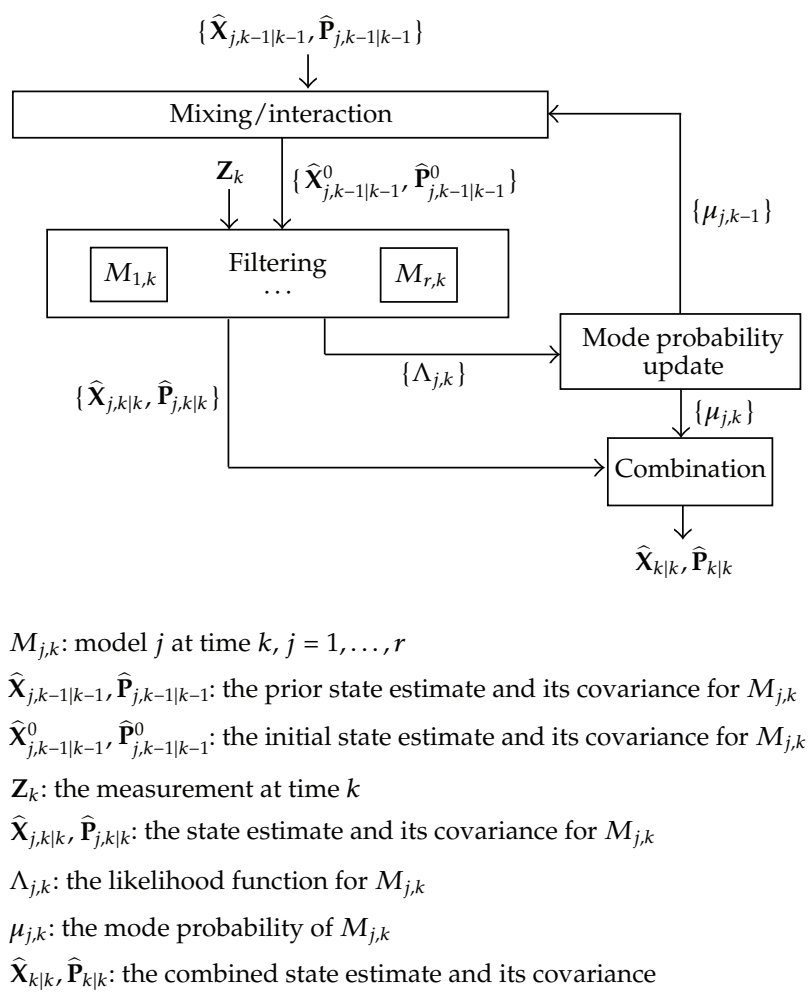

Figure 3: Flow diagram of the IMM method.

The outline of IMMPF algorithm is given below (it assumes that there are $r$ modes).

(1) Interaction. For $M_{j, k}$, compute the initial state estimate and its covariance:

$$
\begin{gathered}
\widehat{\mathbf{X}}_{j, k-1 \mid k-1}^{0}=\sum_{i=1}^{r} \widehat{\mathbf{X}}_{i, k-1 \mid k-1} \mu_{i|j, k-1| k-1,} \\
\widehat{\mathbf{P}}_{j, k-1 \mid k-1}^{0}=\sum_{i=1}^{r} \mu_{i|j, k-1| k-1}\left[\widehat{\mathbf{P}}_{i, k-1 \mid k-1}+\left(\widehat{\mathbf{X}}_{i, k-1 \mid k-1}-\widehat{\mathbf{X}}_{j, k-1 \mid k-1}^{0}\right)\right. \\
\left.\left(\widehat{\mathbf{X}}_{i, k-1 \mid k-1}-\widehat{\mathbf{X}}_{j, k-1 \mid k-1}^{0}\right)^{T}\right],
\end{gathered}
$$

where $\mu_{i|j, k-1| k-1}=\bar{c}_{j}^{-1} p_{i j} \mu_{i, k-1}$ is mixing probability; $\bar{c}_{j}=\sum_{i=1}^{r} p_{i j} \mu_{i, k-1}$ is the normalization constant; $p_{i j}$ is the transition probability for switching from model $i$ to model $j$.

(2) Particle filter. Through the use of the initial state estimate and its covariance from the interaction step, as well as the measurement $\mathbf{Z}_{k}$, model updates for $M_{j, k}$ are performed by computing the state estimate $\widehat{\mathbf{X}}_{j, k \mid k}$, and its covariance $\widehat{\mathbf{P}}_{j, k \mid k}$. The process of particle filter is described as follows: 


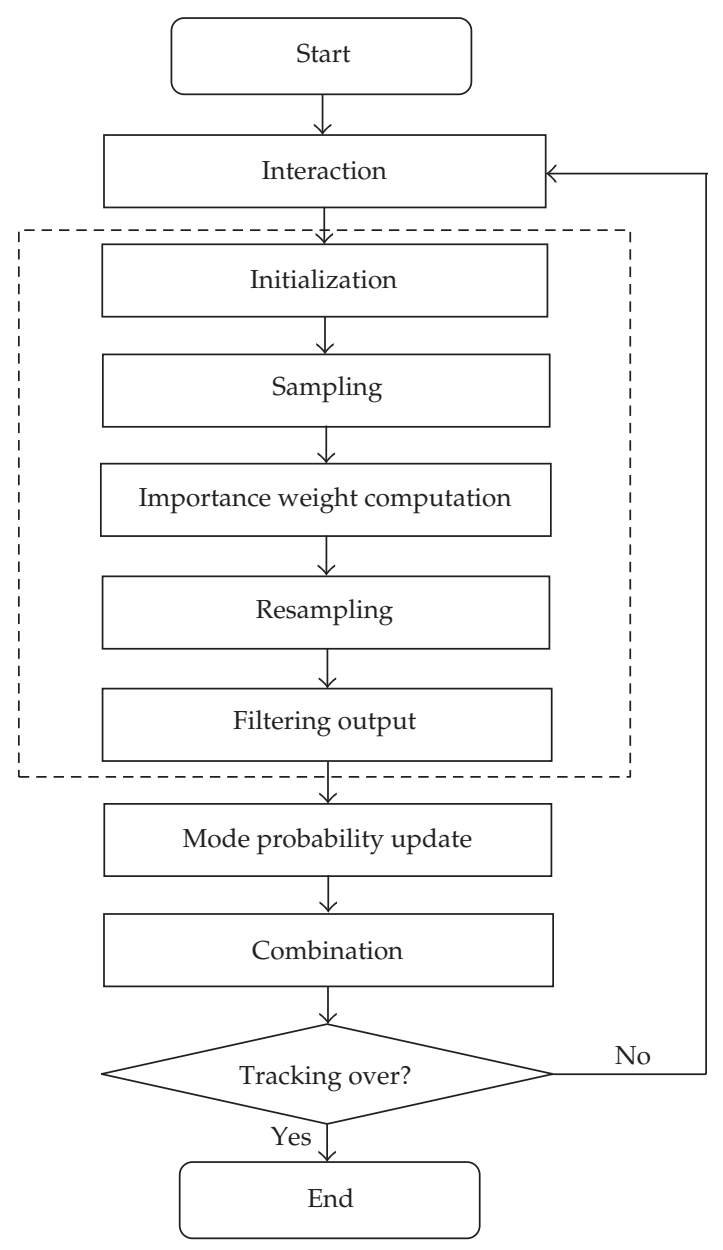

Figure 4: Flowchart of the IMMPF algorithm.

(a) Initialization. Generate samples $\left\{\widehat{\mathbf{X}}_{j, k-1 \mid k-1}^{(i)}\right\}_{i=1}^{N s}$ from the prior probability density function by $N\left(\widehat{\mathbf{X}}_{j, k-1 \mid k-1}^{0}, \widehat{\mathbf{P}}_{j, k-1 \mid k-1}^{0}\right)$. Set initial weights $w_{j, k-1}^{(i)}=1 / N s$.

(b) Sampling. Draw sample $\widehat{\mathbf{X}}_{j, k \mid k}^{(i)}$ from the proposal distribution $q\left(\widehat{\mathbf{X}}_{j, k \mid k}\right.$ । $\left.\widehat{\mathbf{X}}_{j, 0: k-1}, \mathbf{Z}_{j, 1: k}\right)$.

(c) Importance weights computation. Compute and normalize the importance weights:

$$
\begin{gathered}
\widetilde{\boldsymbol{w}}_{j, k}^{(i)}=\widetilde{\boldsymbol{w}}_{j, k-1}^{(i)} \frac{p\left(\mathbf{Z}_{j, k} \mid \widehat{\mathbf{X}}_{j, k \mid k}^{(i)}\right) p\left(\widehat{\mathbf{X}}_{j, k \mid k}^{(i)} \mid \widehat{\mathbf{X}}_{j, k-1 \mid k-1}^{(i)}\right)}{q\left(\widehat{\mathbf{X}}_{j, k \mid k}^{(i)} \mid \widehat{\mathbf{X}}_{j, 0: k-1}^{(i)}, \mathbf{Z}_{j, 1: k}\right)}, \\
w_{j, k}^{(i)}=\frac{\widetilde{\boldsymbol{w}}_{j, k}^{(i)}}{\sum_{i=1}^{N s} \widetilde{\boldsymbol{w}}_{j, k}^{(i)}} .
\end{gathered}
$$


(d) Resampling. Calculate $N_{j, \text { eff }}=\left[\sum_{i=1}^{N s}\left(w_{j, k}^{(i)}\right)^{2}\right]^{-1}$. If $N_{j, \text { eff }}$ is below a user-defined threshold $N_{j, \text { thres, }}$ perform resampling to generate equally weighted particles $\left\{\widehat{\mathbf{X}}_{j, k \mid k^{\prime}}^{(i)}, w_{j, k}^{(i)}\right\}_{i=1}^{N s}$.

(e) Filtering output. Compute the state estimate and its covariance:

$$
\begin{gathered}
\widehat{\mathbf{X}}_{j, k \mid k}=\sum_{i=1}^{N s} w_{j, k}^{(i)} \widehat{\mathbf{X}}_{j, k \mid k^{\prime}}^{(i)} \\
\widehat{\mathbf{P}}_{j, k \mid k}=\sum_{i=1}^{N s} w_{j, k}^{(i)}\left(\widehat{\mathbf{X}}_{j, k \mid k}^{(i)}-\widehat{\mathbf{X}}_{j, k \mid k}\right)\left(\widehat{\mathbf{X}}_{j, k \mid k}^{(i)}-\widehat{\mathbf{X}}_{j, k \mid k}\right)^{T} .
\end{gathered}
$$

(3) Mode probability update. The mode probability of $M_{j, k}$ is computed as

$$
\mu_{j, k}=\frac{1}{c} \Lambda_{j, k} \bar{c}_{j}
$$

where $c=\sum_{i=1}^{r} \Lambda_{i, k} \bar{c}_{i}$. And the likelihood function is calculated as

$$
\Lambda_{j, k}=\mathbb{N}\left(\widetilde{\mathbf{Z}}_{j, k} ; 0, \widehat{\mathbf{S}}_{j, k}\right)
$$

where $\mathbb{N}(\cdot)$ represents Gaussian distribution; $\widetilde{\mathbf{Z}}_{j, k}$ is the filter residual; $\widehat{\mathbf{S}}_{j, k}$ is the corresponding filter residual covariance.

(4) Combination. The combined state estimate and its covariance are computed as:

$$
\begin{gathered}
\widehat{\mathbf{X}}_{k \mid k}=\sum_{i=1}^{r} \mu_{i, k} \widehat{\mathbf{X}}_{i, k \mid k} \\
\widehat{\mathbf{P}}_{k \mid k}=\sum_{i=1}^{r} \mu_{i, k}\left[\widehat{\mathbf{P}}_{i, k \mid k}+\left(\widehat{\mathbf{X}}_{i, k \mid k}-\widehat{\mathbf{X}}_{k \mid k}\right)\left(\widehat{\mathbf{X}}_{i, k \mid k}-\widehat{\mathbf{X}}_{k \mid k}\right)^{T}\right] .
\end{gathered}
$$

\section{Simulation and Results}

To evaluate our proposed method, we design two simulation cases. At first, PF, EKF, and UKF are used to compare the tracking performance for the nonlinear problem of underwater target tracking. Then, the proposed algorithm and PF are applied to a problem on the 3D underwater target tracking, respectively.

\subsection{Performance Evaluations}

We analyze the performance of the presented algorithm and other filtering methods by state estimation errors. The total number of independent simulation runs is $R=50$. Let $\left(x_{k}, y_{k}, z_{k}\right)$ 


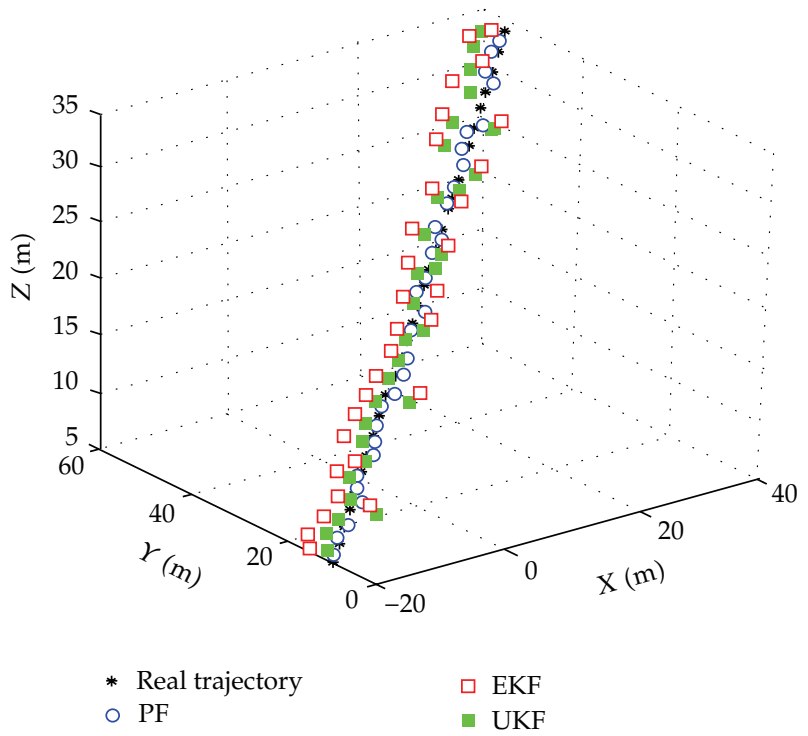

Figure 5: Real and estimated 3D target trajectories obtained with PF, EKF, and UKF.

denote the real position at time $k$, and the corresponding estimated position is $\left(\widehat{x}_{k \mid k}, \widehat{y}_{k \mid k}, \widehat{z}_{k \mid k}\right)$. The tracking errors along the $x$-axis, $y$-axis, and $z$-axis are given as

$$
\begin{aligned}
& E_{x}(k)=\frac{1}{R} \sum_{i=1}^{R}\left|x_{k}-\widehat{x}_{i, k \mid k}\right|, \\
& E_{y}(k)=\frac{1}{R} \sum_{i=1}^{R}\left|y_{k}-\widehat{y}_{i, k \mid k}\right|, \\
& E_{z}(k)=\frac{1}{R} \sum_{i=1}^{R}\left|z_{k}-\widehat{z}_{i, k \mid k}\right|,
\end{aligned}
$$

The root mean square error (RMSE) in the estimation of position is defined as

$$
E(k)=\sqrt{\frac{1}{R} \sum_{i=1}^{R}\left[\left(x_{k}-\widehat{x}_{i, k \mid k}\right)^{2}+\left(y_{k}-\widehat{y}_{i, k \mid k}\right)^{2}+\left(z_{k}-\widehat{z}_{i, k \mid k}\right)^{2}\right]} .
$$

\subsection{Tracking Performance Comparison between PF, EKF, and UKF}

\subsubsection{Simulation Conditions}

In our first experiment, we assume the initial position of the target is $(-20,10,5) \mathrm{m}$, and the initial velocity is $(2,1.5,1) \mathrm{m} / \mathrm{s}$. From 0 to $30 \mathrm{~s}$, it moves at CV. The process noise or measurement noise is $\mathbb{N}\left(0,0.1^{2}\right)$ Gaussian distribution. The sampling interval (time interval between successive scans) is $T=1 \mathrm{~s}$ and the total number of time steps for the duration of tracking a target is $L=30$. 


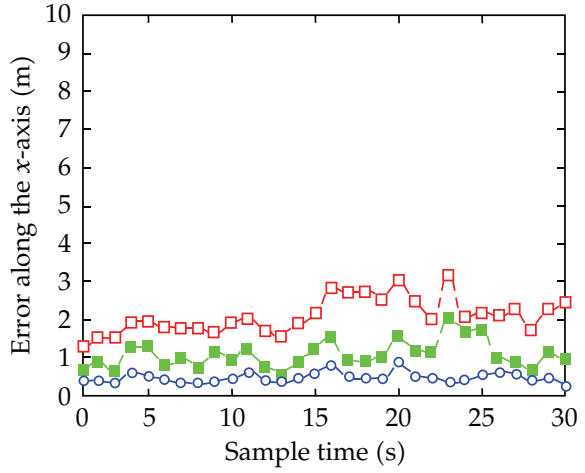

(a)

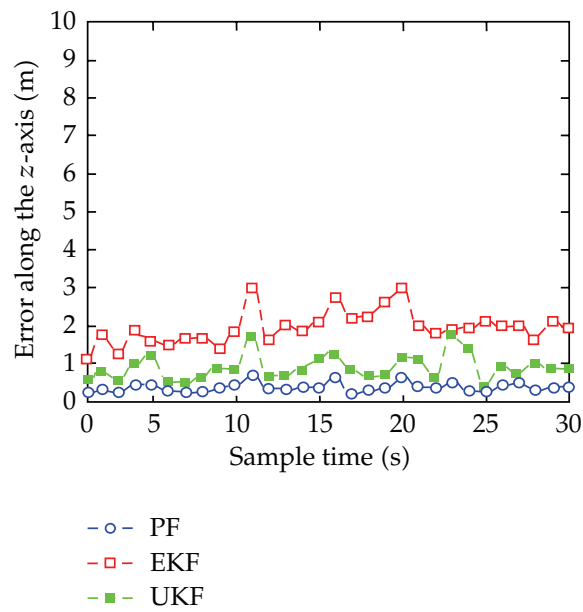

(c)

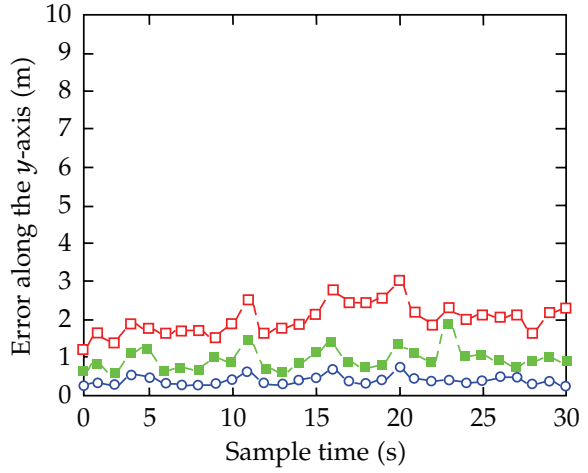

(b)

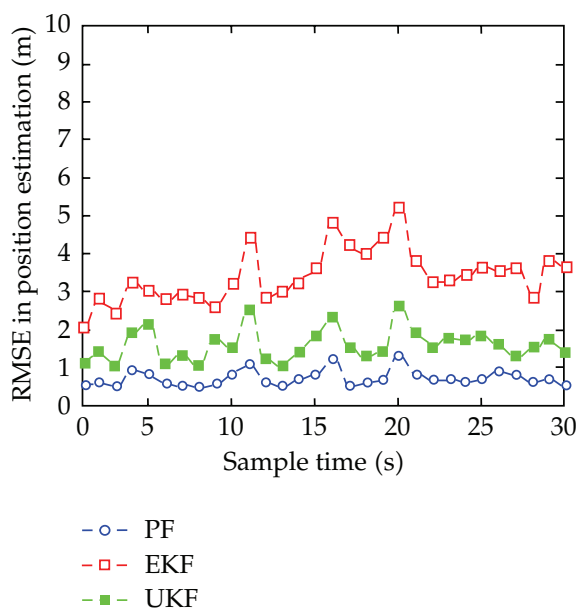

(d)

Figure 6: Tracking performance of PF, EKF, and UKF. (a) The estimation error of the $x$-axis. (b) The estimation error of the $y$-axis. (c) The estimation error of the $z$-axis. (d) RMSE of target position estimation.

\subsubsection{Simulation Results}

Figure 5 shows the real and estimated target trajectories obtained with PF, EKF, and UKF. Figure 6 provides the corresponding target position estimation errors. The comparison results indicate that PF has a better tracking accuracy than EKF and UKF. This is because tracking targets in UWSN is a nonlinear and non-Gaussian problem. EKF uses the first-order Taylor series expansions to approximate nonlinear system functions, and its performance may degrade as the system becomes nonlinear or non-Gaussian. Compared with EKF, UKF transforms the analytic integral operator into an approximate summation operator through a set of deterministic point, so it can improve the tracking precision. Nevertheless, its performance may still be unsatisfactory in multimode and non-Gaussian problems. Moreover, PF is not bounded by linearization models and Gauss assumptions and applies to any nonlinear and non-Gaussian random systems. Therefore, PF is more suitable for underwater target tracking. 


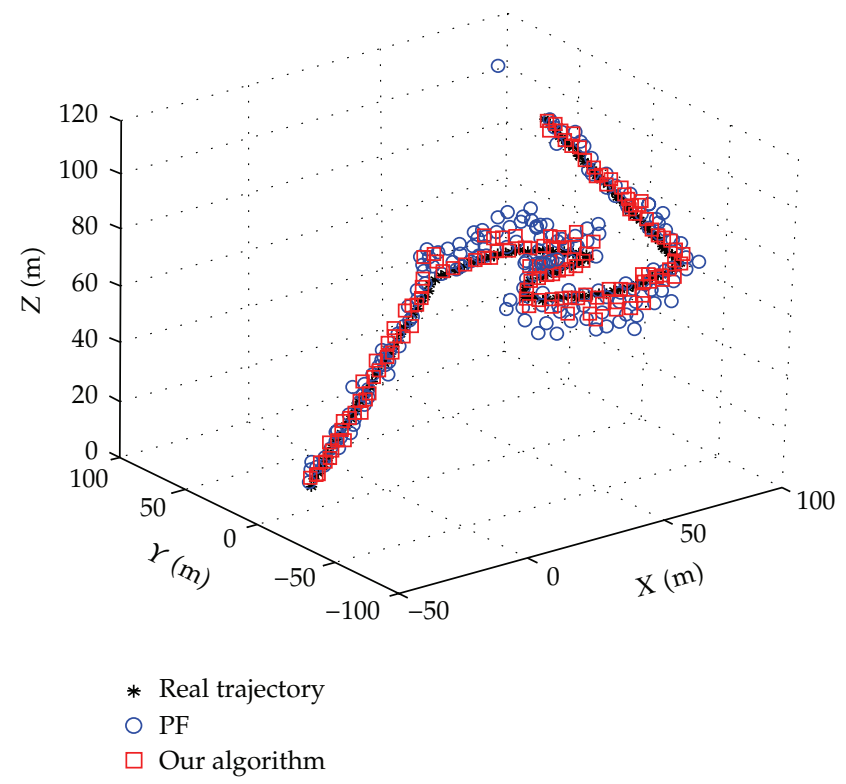

Figure 7: Real and estimated 3D target trajectories obtained with PF and the presented scheme.

\subsection{Tracking Performance Comparison between the Presented Algorithm and PF}

\subsubsection{Simulation Conditions}

The initial state vector is $[-20,2,0,10,1.5,0,5,1,0]^{T}$. From 0 to $40 \mathrm{~s}$, it moves at CV. From 41 to $70 \mathrm{~s}$, it makes a CT with turn rate $\omega=-0.1 \mathrm{rad} / \mathrm{s}$. From 71 to $100 \mathrm{~s}$, it moves at CA. From 101 to $140 \mathrm{~s}$, it makes a CT with turn rate $\omega=0.1 \mathrm{rad} / \mathrm{s}$. From 141 to $170 \mathrm{~s}$, it moves at CV. The process noise or measurement noise is $\mathbb{N}\left(0,0.1^{2}\right)$ Gaussian distribution. The sampling interval is $T=1 \mathrm{~s}$ and the total number of time steps is $L=170$.

Let model 1, model 2, model 3, and model 4 denote CV, CA, and CT with turn rate $\omega=-0.1 \mathrm{rad} / \mathrm{s}, \mathrm{CT}$ with turn rate $\omega=0.1 \mathrm{rad} / \mathrm{s}$, respectively. The transition probability matrix and the initial mode probability are

$$
\left[\begin{array}{cccc}
0.8 & 0.1 & 0.05 & 0.05 \\
0.2 & 0.7 & 0.05 & 0.05 \\
0.15 & 0.05 & 0.75 & 0.05 \\
0.15 & 0.05 & 0.05 & 0.75
\end{array}\right], \quad\left[\begin{array}{llll}
0.8 & 0.1 & 0.05 & 0.05
\end{array}\right]
$$

The initial covariance matrixes are $P_{01}=\operatorname{diag}(9,4,9,4,9,4), P_{02}=\operatorname{diag}(9,4,1,9,4,1,9,4,1)$, $P_{03}=P_{04}=\operatorname{diag}(9,4,0.09,9,4,0.09,9,4,0.09)$.

\subsubsection{Simulation Results}

Figure 7 shows the real and estimated target trajectories of PF and our algorithm, and the corresponding state estimation errors are given in Figure 8. In USWN, the target often does 


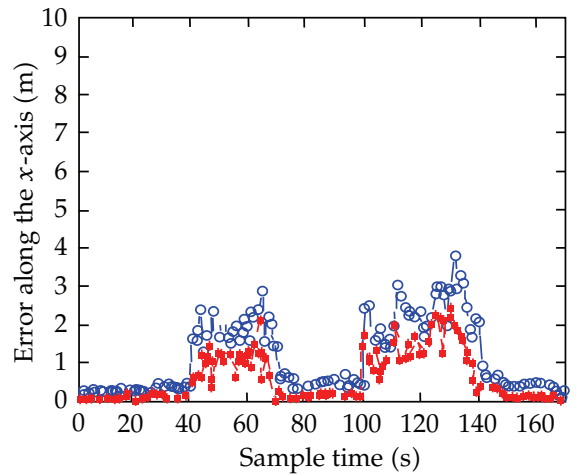

(a)

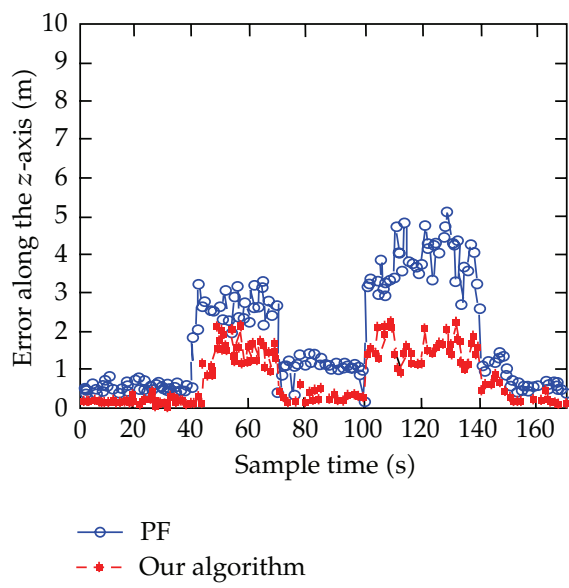

(c)

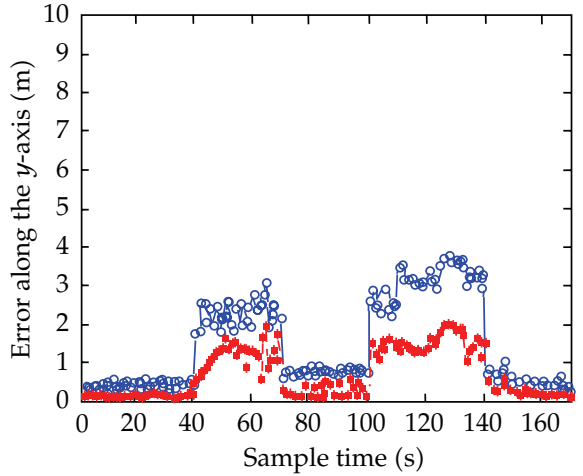

(b)

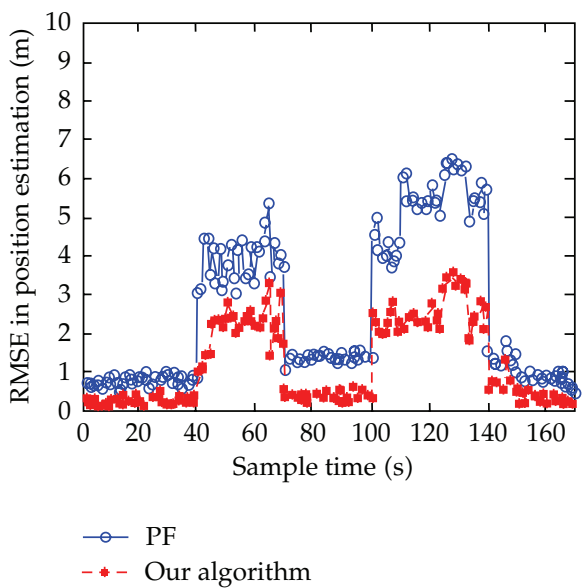

(d)

Figure 8: Tracking performance of PF and the presented scheme. (a) The estimation error of the $x$-axis. (b) The estimation error of the $y$-axis. (c) The estimation error of the $z$-axis. (d) RMSE of target position estimation.

maneuvering motions, which makes the tracking problem intractable. Nevertheless, it can be seen from Figures 7 and 8 that compared with PF, the presented method combining IMM with PF can yield higher tracking precision. In addition, the surges in state estimation errors at the onset and at the termination of a maneuver are also significantly smaller than those for PF.

\section{Conclusions}

This paper focuses on the nonlinear and maneuvering problems for underwater target tracking based on underwater wireless sensor networks. It firstly designs the UWSN architecture for target tracking. Then, to estimate the states of the target, a 3D underwater target tracking algorithm combining interacting multiple model with the particle filter is presented. The simulation results show that compared with PF, EKF, or UKF, the presented method can improve the accuracy and effectiveness of the underwater target tracking. 


\section{Acknowledgments}

This work is supported by the Special Fund for Basic Scientific Research of Central Colleges, Hohai University (Grant no. 2011B09314), the China Postdoctoral Science Foundation funded project (Grant no. 2012M511185) the Jiangsu Planned Projects for Postdoctoral Research Funds (Grant no. 1102069C), and the National Natural Science Foundation of China (Grant No. 61263029).

\section{References}

[1] K. M. Han and H. T. Choi, "Shape context based object recognition and tracking in structured underwater environment," in Proceedings of the IEEE International Conference on Geoscience and Remote Sensing Symposium, pp. 617-620, 2011.

[2] S. Han and $\mathrm{Z}$. Xu, "Passive tracking of maneuvering target based on bearings-frequency of two sonar sensors," in Proceedings of the International Conference on Electronics, Communications and Control, pp. 2174-2177, 2011.

[3] S. K. Rao, K. S. L. Murthy, and K. R. Rajeswari, “Data fusion for underwater target tracking," IET Radar, Sonar and Navigation, vol. 4, no. 4, pp. 576-585, 2010.

[4] S. Y. Chen, "Kalman filter for robot vision: a survey," IEEE Transactions on Industrial Electronics, vol. 59, no. 11, pp. 4409-4420, 2012.

[5] D. Eickstedt, M. Benjamin, H. Schmidt et al., "Adaptive tracking of underwater targets with autonomous sensor networks," Journal of Underwater Acoustics, vol. 56, pp. 465-495, 2006.

[6] H. Shi, W. Wang, N. M. Kwok, and S. Y. Chen, "Game theory for wireless sensor networks: a survey," Sensors, vol. 12, no. 7, pp. 9055-9097, 2012.

[7] S. Chen, W. Huang, C. Cattani, and G. Altieri, "Traffic dynamics on complex networks: a survey," Mathematical Problems in Engineering, vol. 2012, Article ID 732698, 23 pages, 2012.

[8] J. Xue, M. Li, W. Zhao, and S. Chen, "Bound maxima as a traffic feature under DDOS flood attacks," Mathematical Problems in Engineering, vol. 2012, Article ID 419319, 20 pages, 2012.

[9] L. Z. Xu, X. F. Li, and S. X. Yang, “Wireless network and communication signal processing," Intelligent Automation \& Soft Computing, vol. 17, no. 8, article 1019, 2011.

[10] I. F. Akyildiz, D. Pompili, and T. Melodia, "Underwater acoustic sensor networks: research challenges," Ad Hoc Networks, vol. 3, no. 3, pp. 257-279, 2005.

[11] C. H. Yu, K. H. Lee, J. W. Choi, and Y. B. Seo, "Distributed single target tracking in underwater wireless sensor networks," in Proceedings of the SICE Annual Conference, pp. 1351-1356, Japan, August 2008.

[12] G. Isbitiren and O. B. Akan, "Three-dimensional underwater target tracking with acoustic sensor networks," IEEE Transactions on Vehicular Technology, vol. 60, no. 8, pp. 3897-3906, 2011.

[13] E. Kim, S. Lee, C. Kim, and K. Kim, "Bearings-only tracking systems with distributed floating beacons in underwater sensor networks," in Proceedings of the IEEE/IFIP 8th International Conference on Embedded and Ubiquitous Computing, (EUC'10), pp. 311-315, December 2010.

[14] L. Z. Xu, X. F. Ding, X. Wang, G. F. Lv, and F. C. Huang, "Trust region based sequential quasi-Monte Carlo filter," Acta Electronica Sinica, vol. 39, no. 3 A, pp. 24-30, 2011.

[15] S. Y. Chen and Z. J. Wang, "Acceleration strategies in generalized belief propagation," IEEE Transactions on Industrial Informatics, vol. 8, no. 1, pp. 41-48, 2012.

[16] X. F. Ding, L. Z. Xu, X. Wang et al., "Robust visual object tracking using covariance features in QuasiMonte Carlo filter," Intelligent Automation and Soft Computing, vol. 17, no. 5, pp. 571-582, 2011.

[17] X. Wang and Z. Tang, "Modified particle filter-based infrared pedestrian tracking," Infrared Physics and Technology, vol. 53, no. 4, pp. 280-287, 2010.

[18] M. Li, S. C. Lim, and S. Chen, "Exact solution of impulse response to a class of fractional oscillators and its stability," Mathematical Problems in Engineering, vol. 2011, Article ID 657839, 9 pages, 2011.

[19] S. Y. Chen, H. Tong, Z. Wang, S. Liu, M. Li, and B. Zhang, "Improved generalized belief propagation for vision processing," Mathematical Problems in Engineering, vol. 2011, Article ID 416963, 12 pages, 2011.

[20] C. Cattani, "Shannon wavelets for the solution of integrodifferential equations," Mathematical Problems in Engineering, vol. 2010, Article ID 408418, 22 pages, 2010. 
[21] F. Zhang, X. P. Zhou, X. H. Chen, and R. L. Liu, "Particle filter for underwater bearings-only passive target tracking," in Proceedings of the Pacific-Asia Workshop on Computational Intelligence and Industrial Application, (PACIIA '08), pp. 847-851, December 2008.

[22] H. Q. Liu, M. Chitre, and R. Gao, "AUV positioning based on interactive multiple model," in Proceedings of the IEEE Conference on OCEANS, pp. 1-6, May 2010.

[23] C. H. Yu, H. P. Moon, J. W. Choi, Y. B. Seo, T. I. Seo, and E. J. Kim, "Interacting multiple model based distributed target tracking algorithm in UWSNs," in Proceedings of the ICROS-SICE International Joint Conference, (ICCAS-SICE '09), pp. 1752-1759, August 2009.

[24] Y. Boers and J. N. Driessen, "Interacting multiple model particle filter," Proceedings of the IEE Proceedings: Radar, Sonar and Navigation, vol. 150, no. 5, pp. 344-349, 2003.

[25] H. W. Li and J. Wang, "Particle filter for manoeuvring target tracking via passive radar measurements with glint noise," IET Radar, Sonar and Navigation, vol. 6, no. 3, pp. 180-189, 2012.

[26] N. Yang, P. Li, J. Zhang, and Y. Zhu, "Parameter identification of land vehicle location model based on IMMPF," in Proceedings of the 3rd International Conference on Computer Research and Development, (ICCRD '11), pp. 266-269, March 2011.

[27] Z. Zhou, J. H. Cui, and S. L. Zhou, "Localization for large-scale underwater sensor networks," in Proceedings of the 6th international IFIP-TC6 Conference on Ad Hoc and Sensor Networks, Wireless Networks, Next Generation Internet, pp. 108-119, 2007.

[28] B. T. Lee and S. Kim, "Scalable DV-Hop localization for wireless sensor networks," in Proceedings of the 14th Asia-Pacific Conference on Communications, (APCC '08), pp. 1-4, October 2008.

[29] J. Liu, X. Han, M. Al-Bzoor et al., "PADP: prediction assisted dynamic surface gateway placement for mobile underwater networks," in Proceedings of the IEEE Symposium on Computers and Communications, pp. 139-144, 2012.

[30] X. R. Li and V. P. Jilkov, "Survey of maneuvering target tracking. part I: dynamic models," IEEE Transactions on Aerospace and Electronic Systems, vol. 39, no. 4, pp. 1333-1364, 2003.

[31] X. R. Li and V. P. Jilkov, "A survey of maneuvering target tracking-part III: measurement models," in Signal and Data Processing of Small Targets, vol. 4473 of Proceedings of SPIE, pp. 423-446, August 2001.

[32] S. Chen, Y. Wang, and C. Cattani, "Key issues in modeling of complex 3D structures from video sequences," Mathematical Problems in Engineering, vol. 2012, Article ID 856523, 17 pages, 2012.

[33] C. Cattani, S. Chen, and G. Aldashev, "Information and modeling in complexity," Mathematical Problems in Engineering, vol. 2012, Article ID 868413, 4 pages, 2012.

[34] H. A. P. Blom and Y. Bar-Shalom, "Interacting multiple model algorithm for systems with Markovian switching coefficients," IEEE Transactions on Automatic Control, vol. 33, no. 8, pp. 780-783, 1988.

[35] P. H. Foo, "Combining the interacting multiple model method with particle filters for manoeuvring target tracking with a multistatic radar system," IET Radar, Sonar and Navigation, vol. 5, no. 7, pp. 697-706, 2011. 


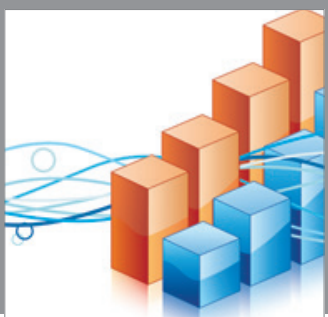

Advances in

Operations Research

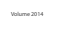

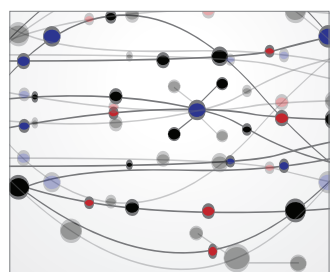

\section{The Scientific} World Journal
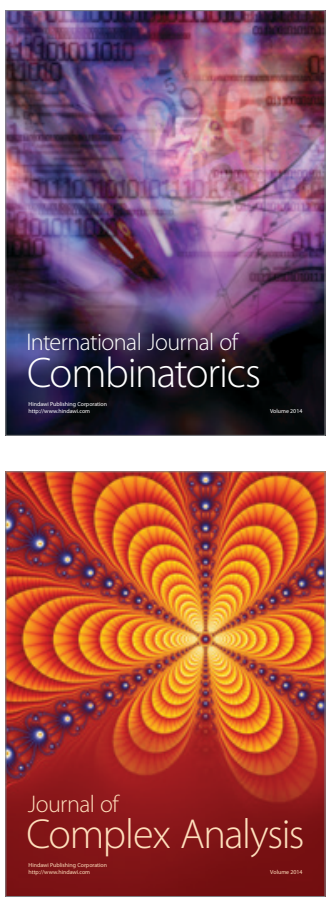

International Journal of

Mathematics and

Mathematical

Sciences
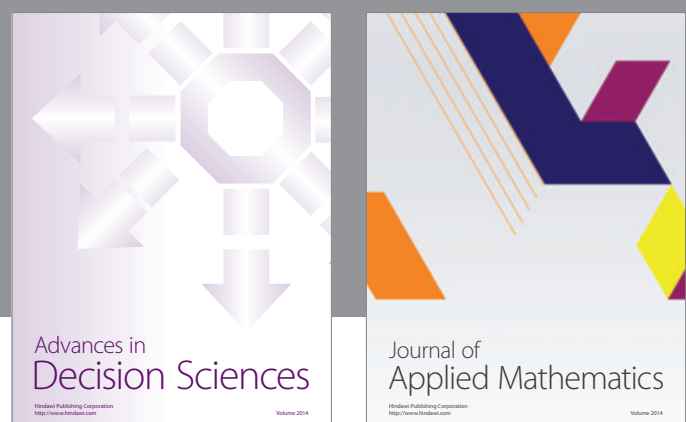

Journal of

Applied Mathematics
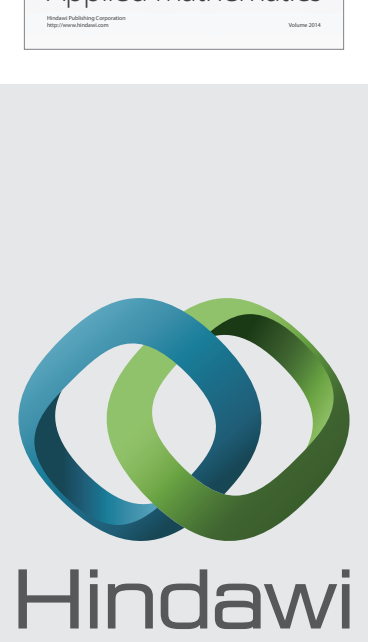

Submit your manuscripts at http://www.hindawi.com
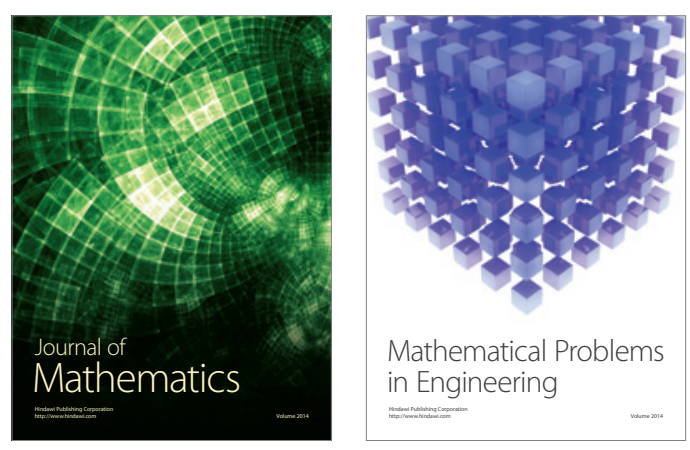

Mathematical Problems in Engineering
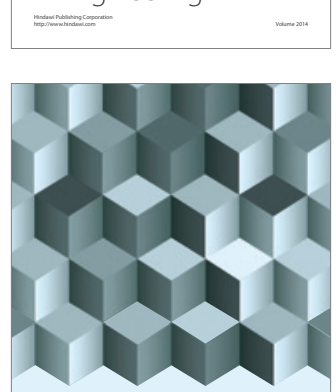

Journal of

Function Spaces
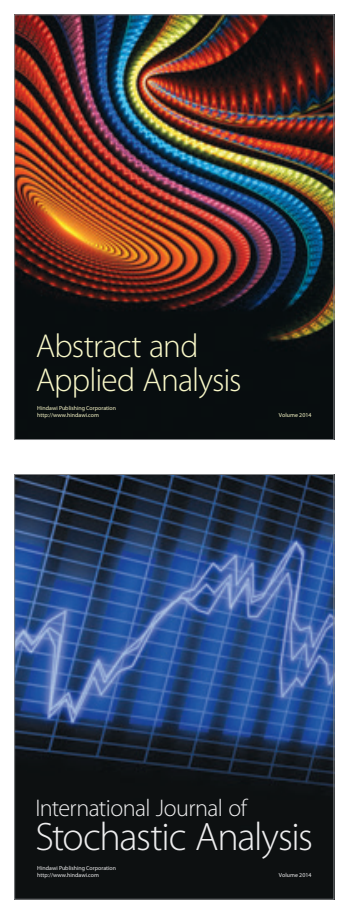

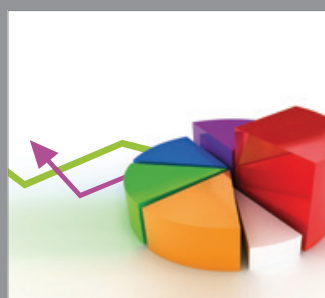

ournal of

Probability and Statistics

Promensencen
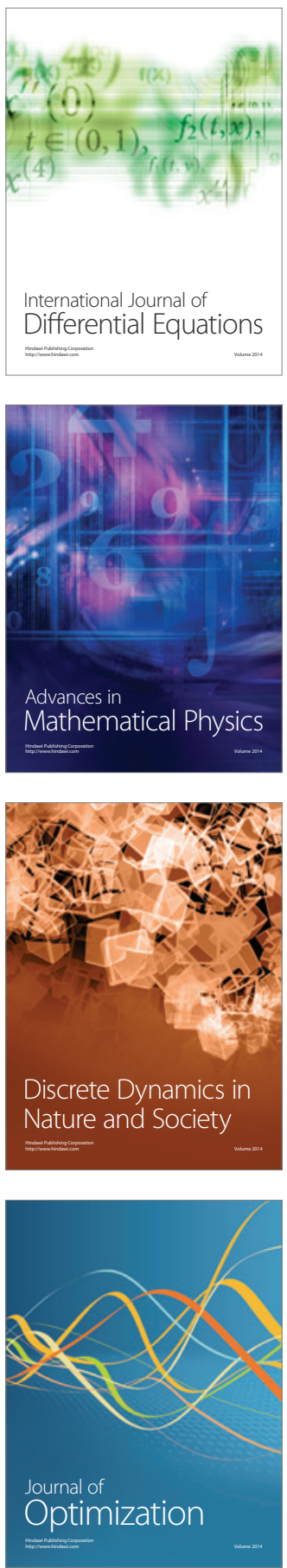